\title{
NOTE ON LEAST-SQUARE APPROXIMATION TO AN ANALYTIC FUNCTION BY POLYNOMIALS, AS MEASURED BY A SURFACE INTEGRAL ${ }^{1}$
}

\author{
J. L. WALSH
}

Relatively satisfactory results on degree of Tchebycheff approximation by polynomials in the closure of a region $D$ bounded by an analytic Jordan curve $C$ to a function analytic in $D$ and satisfying a Lipschitz condition on $C$ were obtained twenty years ago by Curtiss, Sewell and Walsh; these results are analogous to the classical results on approximation by trigonometric sums due in part to Lebesgue, Jackson, S. Bernstein, Montel, and culminating in the theory of de la Vallée Poussin. More recently Walsh and Russell [1] have obtained similar results relating degree of approximation by polynomials in the mean on $C$ to integrated Lipschitz conditions on $C$, analogues of the theory of trigonometric approximation in the mean formulated by Hardy and Littlewood and later developed in detail by Quade. Both of these theories of approximation by polynomials omit the study of degree of approximation by polynomials in the mean on $D$, and the object of the present note is to point the way to such a study; here no analogue exists in the field of pure trigonometric approximation. The present limited methods apply only to least $p$ th power approximation with $p=2$, apply however not only to analytic functions but also to harmonic functions with analogous results, as the writer plans to indicate on another occasion.

A function $f(w)$ analytic in $|w|<1$ is said to be of class $H_{p}$ there $(p \geqq 1)$ provided $\int_{0}^{2 \pi}\left|f\left(r e^{i \theta}\right)\right|^{p} d \theta$ is uniformly bounded for $0<r<1$; if this condition is satisfied, boundary values $f\left(e^{i \theta}\right)$ exist for almost all $\theta$, and $\int_{0}^{2 \pi}\left|f\left(e^{i \theta}\right)\right|^{p} d \theta$ exists. A function $f(w)$ is said to be of class $L_{p}(k, \alpha)$ on $\gamma:|w|=1$ if $f^{(k)}(z)$ is of class $H_{p}$ and if we have

$$
\int_{0}^{2 \pi}\left|f^{(k)}\left(e^{i \theta}\right)-f^{(k)}\left(e^{i(\theta+\phi)}\right)\right|^{p} d \theta \leqq A|\phi|^{p \alpha} ;
$$

here and below we assume $p \geqq 1, k$ a non-negative integer, and (unless otherwise stated) $0<\alpha<1$. Numbers $A$ with or without subscripts represent constants independent of $n$, $w$, and $z$, which may have different meanings in different formulas. If $C$ is an analytic Jordan curve, we say that $f(z)$ is of class $L_{p}(k, \alpha)$ on $C$ if the transform of $f(z)$ is of

\footnotetext{
Presented to the Society, October 25, 1958; received by the editors August 14, 1958

1 Research done (in part) under Contract AF 18(600)-1461 with the U. S. Air Force, Air Research and Development Command.
} 
class $L_{p}(k, \alpha)$ when the interior of $C$ is mapped conformally onto $|w|<1$; it can be shown [1] that this condition is independent of the particular conformal map used, and that the condition (1) on the transform of $f^{(k)}(z)$ is equivalent to the condition

$$
\int_{C}|F(s)-F(s+h)|^{p} d s \leqq A_{1}|h|^{p \alpha},
$$

where $s$ represents arc length on $C, F(s) \equiv f^{(k)}(z)$ on $C$.

With reference to a given Jordan curve $C$, its interior $D$, and a function $f(z)$ analytic in $D$, we denote by $E_{p}=E_{p}(f)$ and $E_{p}^{\prime}=E_{p}^{\prime}(f)$ respectively the non-negative quantities defined by

$$
\begin{aligned}
E_{p}^{p} & =\min \frac{1}{2 \pi} \int_{C}\left|f(z)-p_{n}(z)\right|^{p} d s, \\
E_{p}^{\prime p} & =\min \frac{1}{2 \pi} \iint_{D}\left|f(z)-p_{n}(z)\right|^{p} d S,
\end{aligned}
$$

over all polynomials $p_{n}(z)$ of fixed degree $n$; these minima are known to exist. In particular if $f(z)$ is of class $H_{2}$ in $|z|<1$, or if $|f(z)|^{2}$ is integrable over $|z|<1$ (which is true if $f(z)$ is of class $H_{2}$ ), it is known that we have respectively

$$
\begin{aligned}
E_{2}^{2} & =\frac{1}{2 \pi} \int_{|z|=1}\left|f(z)-s_{n}(z)\right|^{2} d s=\left|a_{n+1}\right|^{2}+\left|a_{n+2}\right|^{2}+\cdots, \\
{E_{2}^{\prime}}^{2} & =\frac{1}{2 \pi} \iint_{|z|<1}\left|f(z)-s_{n}(z)\right|^{2} d S \\
& =\frac{\left|a_{n+1}\right|^{2}}{2(n+2)}+\frac{\left|a_{n+2}\right|^{2}}{2(n+3)}+\cdots,
\end{aligned}
$$

where $s_{n}(z)$ is the partial sum $\sum_{0}^{n} a_{j} z^{j}$ of the Maclaurin development $f(z) \equiv \sum_{0}^{\infty} a_{j} z^{i}$. Convergence of the series $\sum\left|a_{j}\right|^{2}$ or $\sum\left|a_{j}\right|^{2} /(j+1)$ is necessary and sufficient that $f(z)$ be of class $H_{2}$ or square integrable over $|z|<1$.

A principal result of the paper [1] already mentioned is: a necessary and sufficient condition that $f(z)$ be of class $L_{p}(k, \alpha)$ on an analytic Jordan curve $C$ is

$$
E_{p} \leqq A / n^{k+\alpha},
$$$$
n>0 .
$$

The principal result of the present note is

Theorem 1. Let $C$ be an analytic Jordan curve and let $D$ denote the interior of $C$. If the function $f(z)$ is analytic in $D$, a necessary and sufficient condition that $f(z)$ be of class $L_{2}(k, \alpha)$ on $C$ is 


$$
E_{2}^{\prime} \leqq A / n^{k+\alpha+1 / 2} \text {. }
$$

We first establish Theorem 1 for $C$ the unit circle $\gamma:|z|=1$. By inspection of (3) and (4) we have

$$
{E_{2}^{\prime}}^{2} \leqq E_{2}^{2} / 2(n+2) \leqq E_{2}^{2} / n ;
$$

if $f(z)$ is of class $L_{2}(k, \alpha)$ on $C$ we have by (5) the inequality $E_{2}$ $\leqq A_{1} / n^{k+\alpha}$, whence by (7) inequality (6) follows.

To prove the converse we set

$$
f_{1}(z) \equiv \int_{0}^{z} f(z) d z \equiv \sum_{0}^{\infty} \frac{a_{j} z^{j+1}}{j+1}
$$

whence by (3) if $f_{1}(z)$ is of class $H_{2}$

$$
E_{2}^{2}\left(f_{1}\right)=\frac{\left|a_{n}\right|^{2}}{(n+1)^{2}}+\frac{\left|a_{n+1}\right|^{2}}{(n+2)^{2}}+\cdots .
$$

Comparison of (8) with (4) yields (with $n$ replaced by $n-1$ in $E_{2}^{\prime}(f)$ )

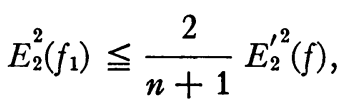

so by our hypothesis (6) the function $f_{1}(z)$ is of class $H_{2}$ and there follows

$$
E_{2}\left(f_{1}\right) \leqq A_{1} / n^{k+\alpha+1} \text {. }
$$

Identification of (10) with (5) shows that $f_{1}(z)$ is of class $L_{p}(k+1, \alpha)$ on $C$, namely that $f(z)$ is of class $L_{p}(k, \alpha)$ on $C$, so Theorem 1 is established for the case that $C$ is $\gamma$.

We outline the proof of Theorem 1 in the case that $C$ is arbitrary; details are similar to those given in [1]. Let $w=\phi(z), z=\psi(w)$ map the interior of $C$ in the $z$-plane onto $|w|<1$; if $f(z)$ is of class $L_{2}(k, \alpha)$ on $C$, then $f[\psi(w)]$ is of class $L_{2}(k, \alpha)$ on $\gamma$, and inequality (6) holds with reference to approximation in $|w|<1$ by the partial sums $s_{n}(w)$ of the Maclaurin development of $f[\psi(w)]$. From (6) there follows

$$
\iint_{D}\left|s_{n}(w)\right|^{2} d S \leqq A_{0},
$$

from which follows $\left[2\right.$, p. 96 , Lemma 2] in turn $\left|s_{n}(w)\right| \leqq A_{1}$ on an arbitrary closed point set $C^{\prime}$ in $|w|<1$; we choose $C^{\prime}$ as a fixed circle $|w|=r(<1)$ independent of $n$. Application of the generalized Bernstein Lemma $[2$, p. 77 , Lemma] establishes

$$
\left|s_{n}(w)\right| \leqq A_{1} R^{n}, \quad R>1,
$$


in the region $|w| \leqq r R$; the precise form of this latter region is of no significance, provided it contains $|w| \leqq 1$ and provided (11) is satisfied. Interpretation of (6) and (11) for the $s_{n}(w)$ now in the $z$-plane enables us to replace the functions $s_{n}[\phi(z)]$ by polynomials in $z$ of suitable degree (the method is due to Curtiss) and thereby to establish (6), where $E_{2}^{\prime}$ now refers to $D$ in the original z-plane.

Conversely, if (6) is given for arbitrary $C$ in the $z$-plane, for some sequence of polynomials $p_{n}(z)$, the method just used to prove (11) establishes the analogue of (11):

$$
\left|p_{n}(z)\right| \leqq A_{1} R^{n}, \quad R>1,
$$

in some region containing $C+D$ in its interior. Interpretation of (6) and (12) in the $w$-plane after the transformation $w=\phi(z)$ enables us to establish the analogue of (6) in the w-plane for suitably chosen polynomials in $w$, from which it follows that $f[\psi(w)]$ is of class $L_{2}(k, \alpha)$ on $|w|=1$, and hence $f(z)$ is of that same class on $C$. Theorem 1 is established.

Theorem 1 is of particular interest in two respects. (i) As with the Tchebycheff measure of approximation and also least $p$ th powers as defined by $E_{p}$, there exists with reference to least squares in the sense of $E_{2}^{\prime}$ a sequence of classes of functions for each $\alpha$, here $L_{2}(0, \alpha)$, $L_{2}(1, \alpha), \ldots$; each class consists of the indefinite integrals of the functions of the preceding class, and each class is completely characterized by a degree of approximation (now of least squares as defined by $E_{2}^{\prime}$ ) involving a power of $n$; these powers (in the denominator) increase by one unit as we move from one class to the succeeding class. (ii) The classes that correspond to $E_{2}^{\prime}$ as measure of approximation are readily expressible in terms of the classes $L_{2}(k, \alpha)$ that correspond to $E_{2}$ as measure of approximation.

It is not surprising that for functions $f(z)$ of class $L_{2}(k, \alpha)$ there should be a difference of $1 / 2$ in the exponents of $n$ in (5) and (6). For instance with $C$ the unit circle $\gamma$ the integral

$$
\frac{1}{2 \pi} \int_{|z|=r}\left|F\left(r e^{i \theta}\right)\right|^{2} d \theta, \quad 0<r \leqq 1,
$$

is a monotonic increasing function of $r$, provided merely $F(z)$ is of class $\mathrm{H}_{2}$. It follows directly from the first equations in (3) and (4) that $2 E_{2}^{\prime 2} \leqq E_{2}^{2}$, and indeed a corresponding inequality follows for $E_{p}$ and $E_{p}$. However, the function $F(z) \equiv\left[f(z)-s_{n}(z)\right] / z^{n+1}$ is also of class $H_{2}$, whence $\left(z=r e^{i \theta}\right)$

$$
\frac{1}{2 \pi} \int_{|z|=r}\left|f(z)-s_{n}(z)\right|^{2} d \theta \leqq \frac{r^{2 n+2}}{2 \pi} \int_{\gamma}\left|f(z)-s_{n}(z)\right|^{2} d \theta,
$$


from which (7) follows. Again, such reasoning is impossible without large modification for least $p$ th power approximation ${ }^{2}$ on $|z|<1$, $p \neq 2$.

The case $\alpha=1$ is exceptional to the foregoing discussion. For this case condition (2) in the definition of class $L_{p}(k, \alpha)$ is to be replaced by the Zygmund condition

$$
\int_{C}|F(s+h)+F(s-h)-2 F(s)|^{p} d s \leqq A_{1}|h|^{p} .
$$

If this replacement is made, inequality (5) remains a necessary and sufficient condition that $f(z)$ be of class $L_{p}(k, 1)$ on $C$; Theorem 1 and its proof remain valid for $\alpha=1$.

Although Theorem 1 gives a discussion of the asymptotic behavior of $E_{2}^{\prime}$ which is complete for the classes $L_{2}(k, \alpha), k \geqq 0$, it does not give a complete characterization of the functions $f(z)$ for which the exponent in (6) is greater than zero but not greater than $1 / 2$, say for

$$
E_{2}^{\prime} \leqq A / n^{\alpha-1 / 2}, \quad 1 / 2<\alpha \leqq 1 .
$$

To fill this gap we introduce as in [1] the Hardy-Littlewood class $L_{2}(-1, \alpha)$ of functions $f(z)$ on $C$ or $\gamma$ defined as the derivatives of the respective functions of class $L_{2}(0, \alpha)$ on $C$ or $\gamma$, and also definable $(0<\alpha<1)$ if $C$ is $\gamma$ as the class of functions analytic in $|z|<1$ for each of which $(0<r<1)$

$$
\frac{1}{2 \pi} \int_{|z|=r}\left|f\left(r e^{i \theta}\right)\right|^{2} d \theta \leqq A /(1-r)^{2(1-\alpha)} .
$$

Theorem 1 is complemented by

TheOREM 2. If $C$ and $D$ satisfy the conditions of Theorem 1, and if $f(z)$ is analytic in $D$, a necessary and sufficient condition that $f(z)$ be of class $L_{2}(-1, \alpha), 1 / 2<\alpha \leqq 1$, on $C$ is (14).

The sufficiency of (14) follows by (8), (9), and (10) when $C$ is $|z|=1$, and otherwise by a conformal map, as in the proof of Theorem 1 . To prove the necessity of (14), we need (thanks to the method used in the proof of Theorem 1) merely establish (14) for the case that $C$ is the unit circle $\gamma$. For an arbitrary function $F(z)$ analytic in

2 Some fragmentary results follow by means of the Hölder inequality. Thus a function of class $L_{p}(k, \alpha)$ on $C$ is by (1) also a function of every $L_{p^{\prime}}(k, \alpha), 1<p^{\prime}<p$. Consequently by Theorem 1 if $f(z)$ is of class $L_{p}(k, \alpha), p>2$, we have (6). If $f(z)$ is of class $L_{2}(k, \alpha)$, we have (6) and therefore $E_{p}{ }^{\prime} \leqq A_{1} / n^{k+\alpha+1 / 2}, 1<p<2$. In the converse direction, the inequality $E_{p}{ }^{\prime} \leqq A / n^{k+\alpha+\delta}, p>2$, implies $E_{2}^{\prime} \leqq A_{1} / n^{k+\alpha+\delta}$, which for $\delta=1 / 2$ implies that $f(z)$ is of class $L_{2}(k, \alpha)$ on $C$; indeed, $E_{p}{ }^{\prime} \leqq A / n^{k+\alpha+\delta}$ with $p>1$ implies $E_{p^{\prime}} \leqq A_{1} / n^{k+o+\delta}$ for $1<p^{\prime}<p$. 
$|z|<1$ and of class $L_{2}$ there, we define the (non-negative) norm by the equation

IVe shall use the

$$
\|F(z)\|^{2}=\frac{1}{2 \pi} \iint_{|z|<1}|F(z)|^{2} d S .
$$

Lemma. If $P(z)$ is a polynomial in $z$ of degree $n$ whose norm on $|z|<1$ is $M$, then we have

$$
\left\|p^{\prime}(z)\right\|^{2} \leqq n(n+1) M^{2} \leqq 2 n^{2} M^{2} .
$$

If we set $P(z) \equiv a_{0}+a_{1} z+\cdots+a_{n} z^{n}$, there follow

$$
\begin{aligned}
M^{2} & =\frac{\left|a_{0}\right|^{2}}{2}+\frac{\left|a_{1}\right|^{2}}{4}+\cdots+\frac{\left|a_{n}\right|^{2}}{2(n+1)}, \\
\left\|P^{\prime}(z)\right\|^{2} & =\frac{\left|a_{1}\right|^{2}}{2}+\frac{2^{2}\left|a_{2}\right|^{2}}{4}+\cdots+\frac{n^{2}\left|a_{n}\right|^{2}}{2 n},
\end{aligned}
$$

and these equations imply (15).

If $f(z)$ is given of class $L_{2}(-1, \alpha)$ on $\gamma$, we use a rectilinear path of integration and set $f_{1}(z) \equiv \int_{0}^{z} f(z) d z$, a function of class $L_{2}(0, \alpha)$ on $\gamma$, and if $S_{n}(z)$ denotes the sum of the first $n+1$ terms of the Maclaurin development of $f_{1}(z)$, we have by Theorem 1

$$
\left\|f_{1}(z)-S_{n}(z)\right\| \leqq A / n^{\alpha+1 / 2} .
$$

The sequence $S_{n}(z)$ converges to $f_{1}(z)$ throughout $|z|<1$, uniformly on $|z| \leqq r(<1)$, and the sequence $S_{n}^{\prime}(z)$ converges to $f(z) \equiv f_{1}^{\prime}(z)$ throughout $|z|<1$, uniformly on $|z| \leqq r<1$. We now use a method of grouping the terms of the series corresponding to the sequence $S_{n}(z)$, due to de la Vallée Poussin. For fixed $n$, we choose $m$ to satisfy $2^{m-1} \leqq n<2^{m}$, and set $(|z|<1)$

$$
f_{1}(z)-S_{n}(z) \equiv\left[S_{2^{m}}(z)-S_{n}(z)\right]+\left[S_{2^{m+1}}(z)-S_{2^{m}}(z)\right]+\cdots .
$$

Special cases of (16) are

$\left\|f_{1}(z)-S_{2^{m}}(z)\right\| \leqq A / 2^{m(\alpha+1 / 2)}, \quad\left\|f_{1}(z)-S_{2^{m+1}}(z)\right\| \leqq A / 2^{(m+1)(\alpha+1 / 2)}$, from which with (16) we deduce

$\left\|S_{2^{m}}(z)-S_{n}(z)\right\| \leqq 2 A / n^{\alpha+1 / 2}, \quad\left\|S_{2^{m+1}}(z)-S_{2^{m}}(z)\right\| \leqq A / 2^{m(\alpha+1 / 2)}$.

The lemma now yields

$$
\begin{aligned}
\left\|S_{2^{m}}^{\prime}(z)-S_{n}^{\prime}(z)\right\| & \leqq 4 A 2^{m} / n^{\alpha+1 / 2} \leqq 4 A 2^{m} / 2^{(m-1)(\alpha+1 / 2)}, \\
\left\|S_{2^{m+1}}^{\prime}(z)-S_{2^{m}}^{\prime}(z)\right\| & \left.\leqq 4 A 2^{m+1} / 2^{m(\alpha+1 / 2}\right), \text { etc. }
\end{aligned}
$$


By differentiation of (17) we have

$$
\begin{aligned}
\| f_{1}^{\prime}(z)- & S_{n}^{\prime}(z) \| \\
& \leqq 8 A / 2^{(m-1)(\alpha-1 / 2)}+8 A / 2^{m(\alpha-1 / 2)}+8 A / 2^{(m+1)(\alpha-1 / 2)}+\cdots \\
& \leqq A_{1} / 2^{(m-1)(\alpha-1 / 2)} \leqq A_{1} / n^{\alpha-1 / 2},
\end{aligned}
$$

where $A_{1}$ is independent of $n$ and $z$, which shows that the first member of (14) is finite and (14) is valid. Theorem 2 is established.

The study of approximation by bounded analytic functions is similar in method and conclusion to the study of approximation by polynomials. The proof of the following theorem is analogous to the proofs of corresponding results (compare [1]) concerning both the Tchebycheff measure of approximation and approximation in the mean on $C$, and is left to the reader:

Theorem 3. Let $C$ be an analytic Jordan curve, let $D$ denote its interior, and let $\Delta$ denote a bounded region containing $D+C$ in its interior. $A$ necessary and sufficient condition that a function $f(z)$ be of class $L_{2}(k, \alpha)$ on $C$ is that there exist functions $f_{n}(z)$ analytic in $\Delta$ satisfying

$$
\begin{aligned}
\iint_{D}\left|f(z)-f_{n}(z)\right|^{2} d S & \leqq A / n^{2 k+2 \alpha+1}, \\
\left|f_{n}(z)\right| & \leqq A_{1} R^{n}, \quad z \text { in } \Delta,
\end{aligned}
$$

where we suppose merely $2 k+2 \alpha+1>0$.

In view of (12) as a consequence of (6), the first part of Theorem 3 is a consequence of the first parts of Theorems 1 and 2; the latter part of Theorems 1 and 2 is a consequence of the latter part of Theorem 3.

\section{REFERENCES}

1. J. L. Walsh and H. G. Russell. Integrated continuity conditions and degree of approximation by polynomials or by bounded analytic functions, Trans. Amer. Math. Soc. (to appear).

2. J. L. Walsh. Interpolation and approximation, Amer. Math. Soc., Colloquium Publications vol. 20, New York, 1935.

HARVARD UNIVERSITY 